\title{
Formação de mestres e doutores no Brasil: uma análise do currículo das pós-graduações em Ciências Biológicas
}

\author{
Training of masters and doctors in Brazil: an analysis \\ of the curriculum of graduate programs in Biological Sciences
}

\begin{abstract}
Larissa Campos Medeiros ${ }^{1}$
${ }^{1}$ Universidade Federal do Rio de Janeiro | Instituto Nacional de Tecnologia | Programa de Química Biológica, área de concentração Educação, Gestão e Difusão em Ciências | Rio de Janeiro | RJ | Brasil. Contato: larissaaps@ gmail.com. ORCID: http://orcid.org/0000-0002$\underline{0425-4943}$
\end{abstract}

\author{
Jacqueline Leta ${ }^{2}$ \\ ${ }^{2}$ Universidade Federal do Rio de Janeiro | Programa de Química Biológica, área de \\ concentração Educação, Gestão e Difusão em Ciências | Rio de Janeiro | RJ | Brasil. Contato: \\ jleta@bioqmed.ufrj.br. ORCID: https://orcid.org/0000-0002-3271-7749
}
Resumo: O presente estudo aborda a questão da formação de mestres e doutores nas Pós-Graduações (PGs) brasileiras. Investiga-se o caso específico das PGs em Ciências Biológicas, apresentando informações sobre a oferta de disciplinas e sobre como se configuram os currículos destes programas. Trata-se de um estudo descritivo e exploratório com abordagem metodológica quali-quantitativa, com dados extraídos de duas fontes principais: a Plataforma de Dados Abertos da CAPES e a Plataforma Sucupira. Os resultados apontaram que as PGs analisadas oferecem disciplinas que direcionam para uma formação do tipo especialista, voltada principalmente para a pesquisa acadêmica e priorizando algum recorte do conhecimento dentro da Biologia, com pouca ou nenhuma oferta de disciplinas que apoiem uma formação mais abrangente.

Palavras-chave: Pós-graduação. Formação de mestres e doutores. Currículo. Ciências Biológicas.

\begin{abstract}
The presente study adresses the issue of training masters and doctors in Brazilian Graduate Programs (GPs). The specific case of GPs in Biological Sciences is investigated, presenting information about the offer of disciplines and how the curriculum are configured in these programs. As a research method, the qualitative and quantitative approach is used through a descriptive and exploratory study, based on the data collected in the following Brazilian databases, 'Plataforma de Dados Abertos da CAPES' and 'Plataforma Sucupira'. The findings pointed out that the GPs analysed offered disciplines that lead to a specialist type of training, mainly focused on academic research and prioritizing a specialized area of technical expertise in the Biology field, with little or no offer of disciplines to support a more broadly training.
\end{abstract}

Keywords: Graduate Programs. Training of masters and doctors. Curriculum. Biological Sciences.

\footnotetext{
- Recebido em: 26 de fevereiro de 2020 - Aprovado em: 1 de abril de 2020
}

DOI: http://dx.doi.org/10.1590/S1414-4077/S1414-40772020000200008

Este é um artigo publicado em acesso aberto sob uma licença Creative Commons

https://creativecommons.org/licenses/by-nc/4.0/ 


\section{Introdução}

Os programas de pós-graduação stricto sensu (PPGs) são os responsáveis pela formação do nível mais alto de educação formal nas Instituições de Ensino Superior (IES), preparando profissionais qualificados para contribuir com o desenvolvimento científico, econômico e social. Muitos autores (BALBACHEVSKY; SCHWARTZMAN, 2010; NETTO, 2018) consideram a Pós-Graduação (PG) no Brasil um caso de sucesso, um sistema nacional sólido, fruto de políticas de Estado que resultaram na expansão em número e diversidade de áreas de atuação, ampliando, de forma relevante, o acesso e a qualificação de pessoal assim como a inserção internacional e o aumento da produção científica do país.

A história deste caso de sucesso inicia-se formalmente em 1951 com a criação da Coordenação de Aperfeiçoamento de Pessoal de Nível Superior (CAPES), que forneceu as diretrizes e desenvolveu mecanismos não apenas para a instituição dos programas, mas também para garantir a qualidade deles. É neste contexto que são elaborados, na década de 1970, os primeiros processos de avaliação dos PPGs.

Embora tal avaliação tenha sido sempre legitimada pela comunidade científica brasileira, são muitas as críticas aos modelos utilizados para avaliar as PGs. Uma das principais críticas é que há um foco excessivo em critérios exclusivamente acadêmicos, em especial os de produtividade científica, deixando de lado outros critérios relevantes para o desenvolvimento da Ciência e Tecnologia (C\&T), como a aplicação do conhecimento adquirido e produzido nas PGs e a formação interdisciplinar e de qualidade dos estudantes (BARATA, 2019; MOREIRA; VELHO, 2008).

Além disso, tal modelo de avaliação estimula a rotina do publish or perish na academia, caracterizado pela busca incessante da produção de artigos científicos como mecanismo principal para vencer na competição por recursos e para ser bem-sucedido na carreira. Esta situação é apontada em alguns estudos como o principal fator desencadeador de problemas sociais, físicos e mentais entre os cientistas, principalmente os mais jovens (DE MEIS et al., 2003; GALDINO et al., 2016; LOUZADA; SILVA FILHO, 2005).

Importante salientar que, ao enfatizar a produção, em especial em periódicos científicos especializados, o modelo de avaliação da CAPES reforça o papel prioritário das pós-graduações na formação de cientistas para a pesquisa básica, que tem aqueles veículos como principal difusor de seus resultados (MEADOWS, 1974). Considerando, no entanto, que a formação de mestres e doutores ocorre principalmente no interior de universidades públicas (concentra mais 
de 76,3\% do total de $\mathrm{PGs}^{1}$ ), espera-se que a diversidade e pluralidade de ações e iniciativas, que caracterizam os ambientes universitários, também estejam incorporadas em suas PGs.

Sobre este ambiente, Mello, Almeida Filho e Ribeiro (2009) apontam que a promoção de modelos de processos educativos, culturais e científicos mais abertos e interdisciplinares atenderá a atual necessidade de formar, além de excelentes cidadãos, indivíduos competentes, criativos, críticos e empreendedores. Spatti, Serafim e Dias (2016) indicam a importância de se buscar formas de promover o espírito crítico e de reflexão nas universidades públicas, segundo os autores "recuperar esse espírito nos currículos, nas práticas pedagógicas, no ambiente das salas de aula, mas também fora delas, é tarefa fundamental" (SPATTI; SERAFIM; DIAS, 2016, p. 352).

Dentre os fatores intrínsecos às PGs que podem propiciar um ambiente plural e diverso à qualificação dos novos mestres e doutores estão o currículo e as disciplinas nele oferecidas, obrigatórias ou não. Ao cursar as disciplinas, os alunos têm contato com informações que podem ser transformadas em conhecimentos e servirão como base para sua capacidade de atuar pessoal e profissionalmente. A importância de se pensar o que precisa ser ensinado se justifica no fato de que a produção de bens, benefícios, produtos ou serviços na sociedade será fruto do trabalho destes alunos (BOTOMÉ; KUBO, 2002)

Se currículo e disciplinas na graduação, ou seja, na etapa inicial de formação profissional são temas amplamente estudados no Brasil, o mesmo não se observa quando a etapa é a pós-graduação. Considerando, portanto, esta lacuna, o presente artigo traz à luz informações sobre como se configuram os currículos das pós-graduações. Para tal, foi selecionada a grande área Ciências Biológicas, considerada estratégica para o desenvolvimento econômico e social do País e responsável por formar profissionais com potencial de atuação em diversos setores econômicos (CAPES, 2010).

Desta forma, o objetivo principal é fornecer um panorama sobre como os currículos dos Programas de Pós-Graduação em Ciências Biológicas estão estruturados a fim de identificar se este ambiente, por meio de uma atividade própria dele (oferta de disciplinas), está alinhado com a concepção de uma formação plural e diversa, preconizada, cada vez mais, por especialistas no campo da educação (PEREIRA; CARNEIRO; GONÇALVES, 2015). A pesquisa apresentada neste trabalho é inédita em duas frentes: ao apresentar um estudo sobre todos os

\footnotetext{
${ }^{1}$ Dados referentes ao ano de 2018, obtidos na planilha BR-CAPES-COLSUCUP-CURSO-2018-2019-10-01 na Plataforma de Dados Abertos da CAPES. Os outros programas (23,7\% dos 4.357) estão distribuídos em Universidades Privadas, Faculdades, Centros Universitários, Institutos de Pesquisa, Centros Federais de Educação Tecnológica e Institutos Federais de Educação, Ciência e Tecnologia.
} 
programas de uma determinada área de avaliação e ao propor uma análise com foco no currículo formal destes.

Para tanto, o artigo apresenta cinco seções, incluindo esta introdução. Na segunda, apresentamos a questão dos currículos e da formação nos PPGs. A terceira seção apresenta detalhes sobre a coleta e análise dos dados, cujos resultados estão expostos na seção seguinte. Por fim, na quinta seção, apontamos algumas considerações finais, contextualizando os resultados e apontando desafios para a formação de pós-graduandos no Brasil.

\section{A questão do currículo nas Pós-Graduações}

Vários estudos apontam o impacto dos currículos para a construção de competências e habilidades nos estudantes do Ensino Superior (ARAÚJO; SILVA; DURÃES, 2018; MARIATT; CHAMPANGNATTE, 2016; MISNI; NIK MAHMOOD; JAMIL, 2020), evidenciando a relevância da organização e atualização do currículo para o alcance dos objetivos de formação profissional e para o estímulo a mudanças no âmbito educacional, social e cultural. Destaca-se, assim, o objetivo das Instituições de Ensino Superior de zelar pelo constante trabalho de melhoria e atualização de suas estruturas curriculares, tal como discutido por Casner-Lotto e Barrington (2006) e Kisgen (2017).

Nas pós-graduações, Taylor (2011) discute o impacto negativo da superespecialização dos programas para a boa formação dos alunos, com currículos cada vez mais fragmentados, desconectados das necessidades da sociedade e do universo não acadêmico. Uma crítica semelhante é lançada por Bosch (2018), que aponta a pressão por publicações científicas como a principal responsável pela redução do tempo dedicado a disciplinas e outras atividades, o que atrapalha ainda mais a percepção dos estudantes de que o trabalho acadêmico está inserido em um contexto mais amplo. Os autores atribuem a ausência de formação crítico-reflexiva e criativa dos discentes - habilidades relevantes para a sociedade atual - como consequência da falta de valorização dada aos currículos.

Botomé e Kubo (2002) chamam atenção para três dimensões que devem ser consideradas ao se criar ou atualizar os currículos das pós-graduações: o tempo (horas dedicadas as disciplinas/atividades), o conteúdo (o que será ensinado) e o desenvolvimento da capacidade de atuar em situações profissionais diversas. Os autores argumentam que mestrados e doutorados precisam formar pessoas para trabalharem com o desconhecido em diversas áreas do conhecimento, e que a responsabilidade específica destes programas é "desenvolver 
capacidade de pessoas para produzirem conhecimento e torná-lo acessível à sociedade" (BOTOMÉ; KUBO, 2002, p. 12).

Para cumprir com este papel, todos os programas precisariam, portanto, desenvolver currículos que estimulassem o desenvolvimento de comportamentos específicos relacionados às funções desempenhadas por cientistas e professores do ensino superior, incluindo a produção de conhecimento, processos de ensino, produção de tecnologia e administração de processos (BOTOMÉ; KUBO, 2002). No entanto, considerando o caso brasileiro, cuja avaliação dos mestrados e doutorados valoriza principalmente a publicação de resultados em artigos e livros, o que se observa, ao longo do tempo, é um movimento no sentido contrário, ou seja, uma redução na carga horária e na dedicação para outras atividades formativas dos futuros profissionais formados nas PGs (BARATA, 2019).

Muito embora pareça haver um consenso da relevância e necessidade de pesquisas que retratem as diferentes dimensões associadas aos currículos dos diferentes níveis educacionais, ainda são escassos os trabalhos nacionais com este foco especial nas PGs. Boa parte da literatura brasileira que trata da temática "Pós-Graduação" aborda questões relacionadas ao perfil dos programas (CERETTA; ANJOS; SIQUEIRA, 2008; SILVA et al., 2019), a avaliação feita pela CAPES (GHENO et al., 2019), a produção científica (SOUZA; REINERT, 2012) e a qualidade dos trabalhos finais desenvolvidos nos mestrados e doutorados (BALZAN, 2012). São raros os trabalhos que analisam aspectos mais formais dos programas (estrutura curricular; regulamentos; proposta do programa etc.) e os que existem são de análises particulares de um determinado programa, muitos deles da área de Educação, como, por exemplo o de Hostins (2013).

Considerando, assim, o cenário apontado anteriormente, o presente estudo apresenta análises que buscam caracterizar as estruturas curriculares de todos os programas de pósgraduação em Ciências Biológicas no Brasil, uma grande área de avaliação da CAPES que será mais bem detalhada nas próximas seções, a fim de revelar se tais programas estão ou não propiciando uma formação abrangente para seus estudantes.

\section{Metodologia}

A pesquisa empírica aqui apresentada é de natureza quali-quantitativa descritiva e exploratória (BRYMAN, 2016). Os dados foram coletados de duas fontes principais, ambas de responsabilidade do Estado brasileiro: a Plataforma de Dados Abertos da CAPES e a Plataforma 
Sucupira (Coleta CAPES). A coleta dos dados foi realizada no período de janeiro e março de 2019.

A organização dos dados foi realizada com o auxílio do programa Excel, onde foi construída uma base de dados nomeada de BR_PPGBiologicas_2013-2016, contendo a identificação dos programas e suas peculiaridades (nome do programa, instituição de ensino superior, ano de criação, área de avaliação da CAPES, nível acadêmico, região do País, natureza jurídica), além das informações sobre as disciplinas de cada programa com suas características (nome da disciplina, ementa, carga horária, créditos e obrigatoriedade).

Os dados coletados na Plataforma de Dados Abertos da CAPES foram utilizados para identificar os programas que pertenciam ao grupo de análise (grande área Ciências Biológicas). A partir desta identificação, foram coletadas manualmente todas as disciplinas ativas dos PPGs, disponíveis no campo "Disciplinas" na Plataforma Sucupira,

A análise das disciplinas baseou-se na técnica de análise de conteúdo (BARDIN, 2006). Após leitura das ementas de todas as disciplinas, foi possível observar que elas se enquadravam em três macrocategorias temáticas, Ciências Naturais e da Saúde, Ciências Humanas e Sociais e Ciências Exatas e da Terra, descritas e exemplificadas da seguinte forma:

$\checkmark$ Ciências Naturais e da Saúde (CNS): disciplinas relacionadas ao estudo das leis e características gerais e fundamentais da natureza e da vida (Ex.: Biologia Molecular; Bases Biológicas de Distúrbios Emocionais; Tópicos em Oncogenética; Ecologia da Polinização e Conservação).

$\checkmark$ Ciências Humanas e Sociais (CHS): disciplinas que abordam aspectos humanos individuais e sociais (Ex.: Didática em Biofísica; Bioética; Tópicos em Filosofia da Ciência; Gestão de Projetos em Inovação; Como Escrever um Artigo Científico).

$\checkmark$ Ciências Exatas e da Terra (CET): disciplinas que abordam análises quantitativas e aplicações destas para informações e estudos sobre o Planeta Terra (Ex.: Métodos Estatísticos em Evolução Molecular; Computação para Ciências Biológicas; Algoritmos para Bioinformática; Geologia).

Após a macrocategorização das disciplinas, foi criada uma grade de subcategorias para o foco principal do conteúdo indicado nas disciplinas. As disciplinas de Ciências Humanas e Sociais foram classificadas em: Comunicação Científica; Docência; Gestão, Política e Economia; Metodologia e Filosofia da Ciência; Ética. As disciplinas de Ciência Exatas e da Terra foram classificadas em: Estatística; Informática; Geografia; Matemática e Física. Já as disciplinas das Ciências Naturais e da Saúde foram categorizadas de acordo com o tipo de dinâmica das aulas: teóricas ou teórico-práticas/estudos de caso.

Todas as análises foram realizadas nos softwares Excel, versão 2016, e Tableau, versão 2019.3. 


\section{Análise dos dados}

Nesta seção, busca-se apresentar e discutir as análises do mapeamento dos programas em Ciências Biológicas no Brasil, e, em seguida, do perfil das disciplinas oferecidas pelos PPGs no período da última avaliação da CAPES.

\subsection{Identificação dos PPGs em Ciências Biológicas no Brasil}

Na avaliação quadrienal 2013-2016, a CAPES organizava os PPGs em três Colégios, subdivididos em nove Grandes Áreas de Avaliação. Inserida no Colégio Ciências da Vida está a grande área Ciências Biológicas, que por sua vez é subdivida em quatro Áreas de Avaliação: Ciências Biológicas I; Ciências Biológicas II; Ciências Biológicas III e Biodiversidade.

O quadro 1 apresenta alguns exemplos de programas ativos atualmente e inseridos nestas áreas de avaliação.

\section{Quadro 1 - Exemplos de programas pertencentes às quatro áreas de avaliação das Ciências}

\section{Biológicas, 2019}

\begin{tabular}{|c|c|}
\hline $\begin{array}{l}\text { Área de avaliação da } \\
\text { Capes }\end{array}$ & Exemplos de Programas de Pós-Graduação \\
\hline Biodiversidade & $\begin{array}{l}\text { Ecologia; Biodiversidade Animal; Oceanografia; Botânica; } \\
\text { Biologia Comparada; Sistemas Aquáticos Tropicais. }\end{array}$ \\
\hline C. Biológicas I & $\begin{array}{l}\text { Genética; Biologia Celular e Molecular; Biociências; } \\
\text { Biologia Humana e Experimental; Biotecnologia; } \\
\text { Bioinformática. }\end{array}$ \\
\hline C. Biológicas II & $\begin{array}{l}\text { Bioquímica; Fisiologia e Farmacologia; Inovação } \\
\text { Tecnológica e Biofarmacêutica; Neurociências; } \\
\text { Morfotecnologia. }\end{array}$ \\
\hline C. Biológicas III & $\begin{array}{l}\text { Microbiologia e Parasitologia; Imunologia e Inflamação; } \\
\text { Patologia; Biologia Experimental; Biologia Parasitária. }\end{array}$ \\
\hline
\end{tabular}

Fonte: Elaborado pelas autoras com dados da Plataforma de Dados Abertos da Capes, 2019.

A partir dos dados coletados na Plataforma de Dados Abertos CAPES, no último quadriênio (2013-2016), foram identificados 312 PPGs na grande área Ciências Biológicas, distribuídos em 102 IES, em todas as regiões do Brasil, sendo que apenas dezessete destes são programas particulares de natureza jurídica privada. Do total de PPGs, 226 deles se dedicam à formação de mestres e doutores; o restante, inclui programas que formam apenas mestres, 17 mestres profissionais e 2 exclusivamente doutores. Considerando o reduzido número de programas voltados somente para a formação de doutor, decidimos somá-lo ao nível mestrado/doutorado. Assim, este nível abrange 228 programas, representando 73,1\% do total. 
A fim de melhor descrever os 312 programas, apresentamos na Tabela 1 a distribuição destes segundo a sua localização regional, o nível e a área de avaliação em Ciências Biológicas.

Uma primeira observação diz respeito à não uniformidade na distribuição dos programas no território nacional, com uma concentração maior de programas em todos os níveis na região Sudeste, que juntos somam 147 programas, ou seja, 47,1\% do total. Essa constatação é menos evidente, no entanto, quando observamos a distribuição dos programas em Biodiversidade. Esta área possui um percentual menor de concentração no Sudeste quando comparado as outras e uma distribuição mais uniforme dos programas por todas as regiões do país, com exceção do mestrado profissional, que ainda conta com poucos PPGs. Destaca-se também o limitado número de PPGs nas regiões Centro-Oeste e Norte, com uma concentração muito menor de programas do que as outras regiões.

Tabela 1 - Distribuição dos programas por região, nível e área de avaliação, 2017

\begin{tabular}{|c|c|c|c|c|c|c|c|c|}
\hline \multirow{2}{*}{ Nivel } & \multirow[b]{2}{*}{ Área de Avaliação } & \multicolumn{7}{|c|}{ Região } \\
\hline & & $\mathbf{N}$ & NE & $\mathrm{CO}$ & SE & $\mathrm{S}$ & MC & Total \\
\hline \multirow[t]{5}{*}{$\mathbf{M}$} & Biodiversidade & 5 & 14 & 7 & 13 & 5 & & 44 \\
\hline & Ciências Biológicas I & & 4 & 1 & 3 & 2 & & 10 \\
\hline & Ciências Biológicas II & 1 & 3 & & 2 & 2 & & 8 \\
\hline & Ciências Biológicas III & & 4 & 1 & & & & 5 \\
\hline & Total & 6 & 25 & 9 & 18 & 9 & & 67 \\
\hline \multirow[t]{5}{*}{ M/D } & Biodiversidade & 10 & 17 & 7 & 41 & 17 & & 92 \\
\hline & Ciências Biológicas I & 2 & 6 & 3 & 30 & 9 & & 50 \\
\hline & Ciências Biológicas II & 1 & 8 & 1 & 30 & 15 & 2 & 57 \\
\hline & Ciências Biológicas III & 4 & 3 & 3 & 16 & 3 & & 29 \\
\hline & Total & 17 & 34 & 14 & 117 & 44 & 2 & 228 \\
\hline \multirow[t]{5}{*}{ MP } & Biodiversidade & & 1 & & 3 & 1 & & 5 \\
\hline & Ciências Biológicas I & 1 & & & 2 & & & 3 \\
\hline & Ciências Biológicas II & & 1 & & 6 & 1 & & 8 \\
\hline & Ciências Biológicas III & & & & 1 & & & 1 \\
\hline & Total & 1 & 2 & & 12 & 2 & & 17 \\
\hline \multicolumn{2}{|c|}{ Total - Grande Área } & 24 & 61 & 23 & 147 & 55 & 2 & 312 \\
\hline \multicolumn{9}{|c|}{ Soma n" de PPG's } \\
\hline
\end{tabular}

Fonte: Elaborado pelas autoras com dados da Plataforma de Dados Abertos da Capes, 2019.

Nota: M- Mestrado; M/D - Doutorado; MP - Mestrado Profissional; N- Norte; NE- Nordeste; CO- CentroOeste; S - Sul; MC- Multicêntrico

A segunda observação diz respeito aos dois programas multicêntricos (MC) nas Ciências Biológicas II. Estes programas possibilitam a formação de alunos em diferentes 
regiões do Brasil, por meio de convênios realizados com diversas IES, que já possuem pesquisadores com competência para atuar na área, mas que ainda não tem condições de implementar um PPG. Desta forma, estimula-se a criação de novos grupos de pesquisa e a formação de profissionais em diversas regiões do país. Atualmente nas Ciências Biológicas os MC são limitados as áreas de Fisiologia e Biologia Molecular e Bioquímica. A criação de mais programas com este perfil poderia contribuir para uma diminuição do contraste na oferta de formação pós-graduada em diversas áreas nas diferentes regiões do País.

\subsection{Categorias temáticas das disciplinas ofertadas pelos programas}

Para identificar e caracterizar o perfil das estruturas curriculares oferecidas aos pósgraduandos, além das semelhanças e diferenças na oferta de disciplinas dentro das diferentes áreas de avaliação e de cada programa individualmente, as ementas das 13.407 disciplinas (eletivas e obrigatórias) foram submetidas a análise de conteúdo, sendo estas organizadas em três macrocategorias temáticas: Ciências Naturais e da Saúde (CNS), Ciências Humanas e Sociais (CHS) e Ciências Exatas e da Terra (CET).

O gráfico 1 apresenta o perfil de distribuição de todas as disciplinas segundo a macrocategoria temática (CNS, CHS e CET) em cada uma das quatro áreas de avaliação, organizadas pelo nível dos PPGs. Observa-se um perfil semelhante entre as áreas, com uma predominância absoluta de disciplinas classificadas como CNS ( $\mathrm{n}=10.734$ em todas áreas) e ocorrência menor de disciplinas CHS $(n=1.633)$ e CET $(n=1.041)$, o que representa $80 \%, 12 \%$ e $8 \%$ respectivamente.

Importante destacar que as disciplinas de CET nas áreas de avaliação Biodiversidade e Ciências Biológicas I representam, proporcionalmente, o dobro ou mais das outras duas áreas. Essa diferença é consequência do grande número de disciplinas relacionadas à estatística, em especial nos programas de Ecologia - presentes na área de avaliação Biodiversidade - e ao quantitativo de disciplinas de informática nos programas de Biologia Molecular e Bioinformática - inseridos na área de avaliação Ciências Biológicas I, o que será mais bem detalhado na próxima seção.

Ao analisar área e nível em conjunto, os perfis de distribuição das macrocategorias são semelhantes para a maioria dos programas, com exceção dos mestrados profissionais da Biodiversidade e Ciências Biológicas II, que apresentam uma proporção maior de disciplinas CHS, e das Ciências Biológicas I, que apresentam uma maior proporção de CNS, o que será mais bem detalhado na análise dos grupos de disciplinas dentro das macrocategorias. 
Gráfico 1 - Distribuição quantitativa e percentual das disciplinas nas categorias temáticas segundo a área e avaliação da Capes e nível do PPG, 2019

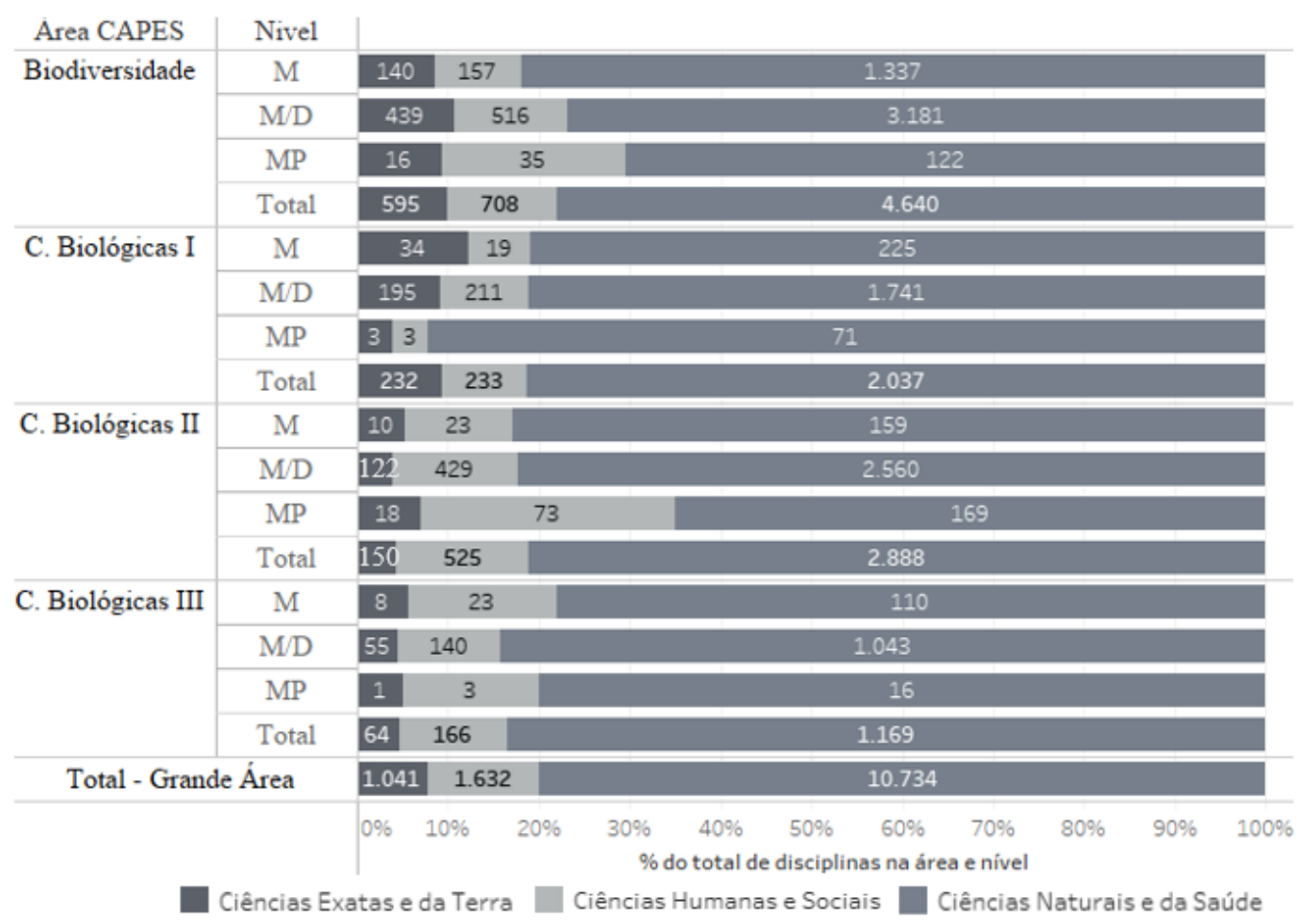

Fonte: Elaborado pelas autoras com dados da Plataforma Sucupira, 2019.

Nota: M- Mestrado; M/D - Mestrado e Doutorado; MP - Mestrado Profissional

Ao analisar a macrocategorização das disciplinas dos programas individualmente, foram observadas algumas exceções (2,5\% dos programas) que destoavam da distribuição percentual apresentada pela Grande Área das Ciências Biológicas, como, por exemplo, o Programa de Bioinformática (C. Biológicas I/MD) da Universidade de São Paulo, que apresentou proporção de $25,5 \%$ das disciplinas na macrocategoria CNS, $2,1 \%$ na CHS e $72,4 \%$ na CET, ou o Programa de Inovação Tecnológica e Propriedade Intelectual (C. Biológicas II /MD) da Universidade Federal de Minas Gerais, que apresentou a distribuição de 31,4\% para CNS e 68,6\% CHS, e nenhuma disciplina categorizada na CET. Nesta análise individual, não foram observadas diferenças na distribuição das macrocategorias que pudessem ser relacionadas com a região do país, natureza administrativa dos programas (públicos ou privados), ano de criação ou nota da CAPES.

A observação das disciplinas obrigatórias dos 312 programas (gráfico 2), ou seja, disciplinas compulsórias para obtenção do título final ao concluir o PPG, indicou uma redução na fração de disciplinas classificadas como CNS, que passam a representar 803 disciplinas (62,4\% do total de 1.286), um aumento das classificadas como CHS (380 disciplinas; 29,5\%) e 
uma proporção semelhante a análise anterior das disciplinas CET $(103 ; 8,1 \%)$ nas áreas de avaliação.

\section{Gráfico 2 - Distribuição quantitativa e percentual das disciplinas obrigatórias nas categorias} temáticas, segundo a área e avaliação da CAPES e nível do PPG, 2019

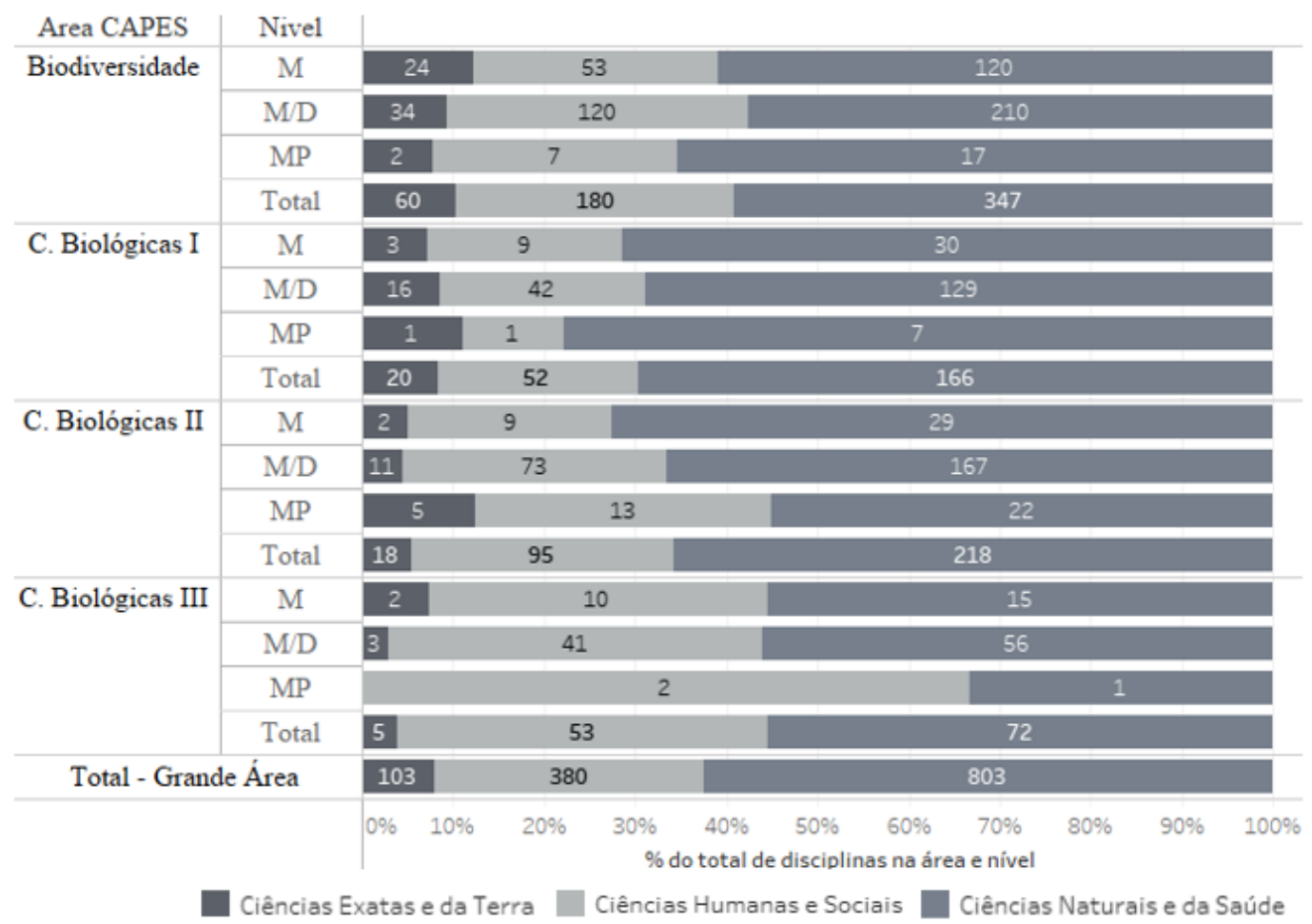

Fonte: Elaborada pelas autoras com dados da Plataforma Sucupira, 2019.

Assim como apontado na análise anterior observou-se uma maior proporção de disciplinas da CET nas áreas Biodiversidade e Ciências Biológicas I. Importante constatar que as disciplinas CHS foram proporcionalmente mais frequentes entre as obrigatórias da área de avaliação Ciências Biológicas III, em especial no Mestrado Profissional, que é composto por apenas um programa.

Os resultados apontaram que, com raras exceções, houve um domínio do conteúdo específico das Ciências Biológicas, situado dentro da macrocategoria Ciências Naturais e da Saúde. Ao realizar a mesma análise, considerando apenas as disciplinas obrigatórias, o conteúdo específico continuou em maior proporção do que os demais, mas com menor predominância do que quando considerado a totalidade de disciplinas.

Para representar com mais clareza o tipo de conteúdo oferecido dentro das três macrocategorias, foi realizada uma nova categorização que é apresentada na próxima subseção. 


\subsection{Grupos de disciplinas mais frequentes dentro das macrocategorias temáticas}

Para entender melhor o perfil das disciplinas das Ciências Biológicas ofertadas nos PPGs, foi realizada uma nova categorização dentro das macrocategorias. Na macrocategoria CNS, as disciplinas foram classificadas de acordo com o tipo de dinâmica de aulas, tendo como subcategorias: Teórico-práticas (incluem disciplinas práticas, de estudo de caso e/ou de trabalho de campo) e Teóricas (disciplinas cujas ementas não especificaram alguma etapa prática). Com esta categorização foi constatado que mais de $90 \%$ (9.649) das disciplinas foram registradas como Teóricas.

Para as disciplinas situadas nas macrocategorias CHS e CET, pertencentes a áreas do conhecimento diferentes das de conteúdo específico das Ciências Biológicas (CNS), foram encontradas uma maior diversidade de tópicos de conteúdo. Na categoria CHS, as disciplinas foram classificadas nas subcategorias: Docência; Comunicação Científica; Gestão, Política e Economia; Metodologia e Filosofia da Ciência; Ética. Já na categoria CET, as subcategorias foram: Estatística e Análise de Dados; Informática; Geografia; Matemática e Física.

O resultado da distribuição das subcategorias de CHS e CET, conforme a áreas de avaliação e o nível dos programas, é apresentado na tabela 2, onde pode ser observado um mapa de calor com a distribuição de todas as disciplinas nas subcategorias. Os números representam o quantitativo de disciplinas nas áreas e níveis indicados na tabela e a saturação de cores de cada célula indica o percentual da subcategoria nestes PPGs (mesmo nível e área).

Observa-se que dentro das disciplinas de todos os programas, há uma maior oferta de disciplinas com conteúdos relacionados: (a) à Docência que somam 608, ou seja 22,7\% do total de disciplinas 37,2\% do total de disciplinas da CHS e (b) à Estatística e Análise de Dados que somam 565, ou seja, $21,1 \%$ do total de disciplinas e 54,2\% de disciplinas da CET.

A análise das subcategorias pelo nível mostra que, entre as disciplinas de CHS, há uma maior oferta de disciplinas relacionadas à Docência e em seguida de Comunicação Científica nos programas acadêmicos, e de disciplinas de Gestão, Política e Economia nos Mestrados Profissionais. Já para as disciplinas de CET, há um domínio de Estatística seguido por disciplinas relacionadas à Informática para todos os níveis. 
Tabela 2 - Distribuição de todas as disciplinas nas subcategorias, 2019

\begin{tabular}{|c|c|c|c|c|c|c|}
\hline & \multirow[b]{2}{*}{ Subcategoria } & \multicolumn{5}{|c|}{ Área CAPES } \\
\hline & & Biodiversidade & $\begin{array}{c}\text { Ciências } \\
\text { Biológicas I }\end{array}$ & $\begin{array}{c}\text { Ciências } \\
\text { Biológicas II }\end{array}$ & $\begin{array}{c}\text { Ciências } \\
\text { Biológicas III }\end{array}$ & Total \\
\hline \multirow{5}{*}{ 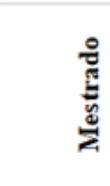 } & Docência & 53 & 8 & 12 & 8 & 81 \\
\hline & Comunicação & 38 & 5 & 3 & 3 & 49 \\
\hline & Ética e Biossegurança & 2 & 3 & 6 & 6 & 17 \\
\hline & Gestão \& Política & 26 & 1 & & & 27 \\
\hline & Metodologia & 31 & & 2 & 5 & 38 \\
\hline \multirow{5}{*}{ 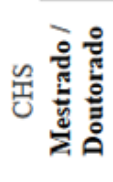 } & Docência & 181 & 93 & 177 & 61 & 512 \\
\hline & Comunicação & 137 & 46 & 90 & 24 & 297 \\
\hline & Ética e Biossegurança & 9 & 32 & 66 & 26 & 133 \\
\hline & Gestão \& Política & 105 & 21 & 42 & 11 & 179 \\
\hline & Metodologia & 85 & 22 & 55 & 18 & 180 \\
\hline \multirow{5}{*}{ 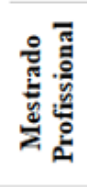 } & Docência & 1 & & 14 & & 15 \\
\hline & Comunicação & 4 & 1 & 9 & & 14 \\
\hline & Ética e Biossegurança & 4 & 1 & 12 & 1 & 18 \\
\hline & Gestão \& Política & 23 & 1 & 27 & 2 & 53 \\
\hline & Metodologia & 9 & & 10 & & 19 \\
\hline \multirow{4}{*}{ 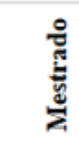 } & Estatística & 106 & 12 & 10 & 5 & 133 \\
\hline & Física e Matemática & 1 & & & & 1 \\
\hline & Geografia & 25 & & & & 25 \\
\hline & Informática & 9 & 24 & & 3 & 36 \\
\hline \multirow{4}{*}{ 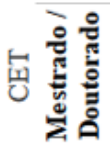 } & Estatística & 242 & 63 & 71 & 31 & 407 \\
\hline & Física e Matemática & 5 & 15 & 20 & 2 & 42 \\
\hline & Geografia & 131 & 11 & & 1 & 143 \\
\hline & Informática & 55 & 105 & 34 & 22 & 216 \\
\hline \multirow{4}{*}{ 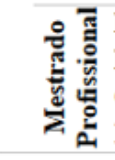 } & Estatística & 11 & 2 & 11 & 1 & 25 \\
\hline & Fisica e Matemática & & & 3 & & 3 \\
\hline & Geografia & 5 & & & & 5 \\
\hline & Informática & 2 & 1 & 2 & & 5 \\
\hline & + & 1.300 & 467 & 676 & 230 & 2.673 \\
\hline & & & & & \multicolumn{2}{|c|}{ \% da subcategorização } \\
\hline & & & & & $0,71 \%$ & 100,0 \\
\hline
\end{tabular}

Fonte: Elaborada pelas autoras com dados da Plataforma Sucupira, 2019.

A análise com foco nas áreas revela que na Biodiversidade, Ciências Biológicas I e Ciências Biológicas II, observa-se uma maior oferta de disciplinas de Docência e Comunicação Científica, enquanto na área Ciências Biológicas III há uma proporção maior de disciplinas de Docência e Ética e Biossegurança. Já entre as disciplinas de CET, há um predomínio de disciplinas de Estatística e Geografia na área de Biodiversidade, ofertadas principalmente nos programas relacionados à Ecologia e Biogeografia. Na área Ciências Biológicas I há um domínio de disciplinas de Informática, muito ofertadas nos programas relacionados à Genética e Bioinformática, e em seguida há uma maior oferta de disciplinas de Estatística.

Ao analisar as disciplinas obrigatórias (Tabela 3), constata-se a ausência de algumas subcategorias, antes presentes nas análises de todas as disciplinas Nota-se também que as que apresentam conteúdo voltados à Docência são ainda mais frequentes neste segmento: somam 255, ou seja 52,6\% do total de disciplinas obrigatórias e 66,4\% das obrigatórias da CHS. Após 
estas disciplinas, as relacionadas a Estatística são as mais recorrentes, representando 16,9\% do total de obrigatórias e $82 \%$ das obrigatórias da CET.

Tabela 3 - Distribuição das disciplinas obrigatórias nas subcategorias, 2019

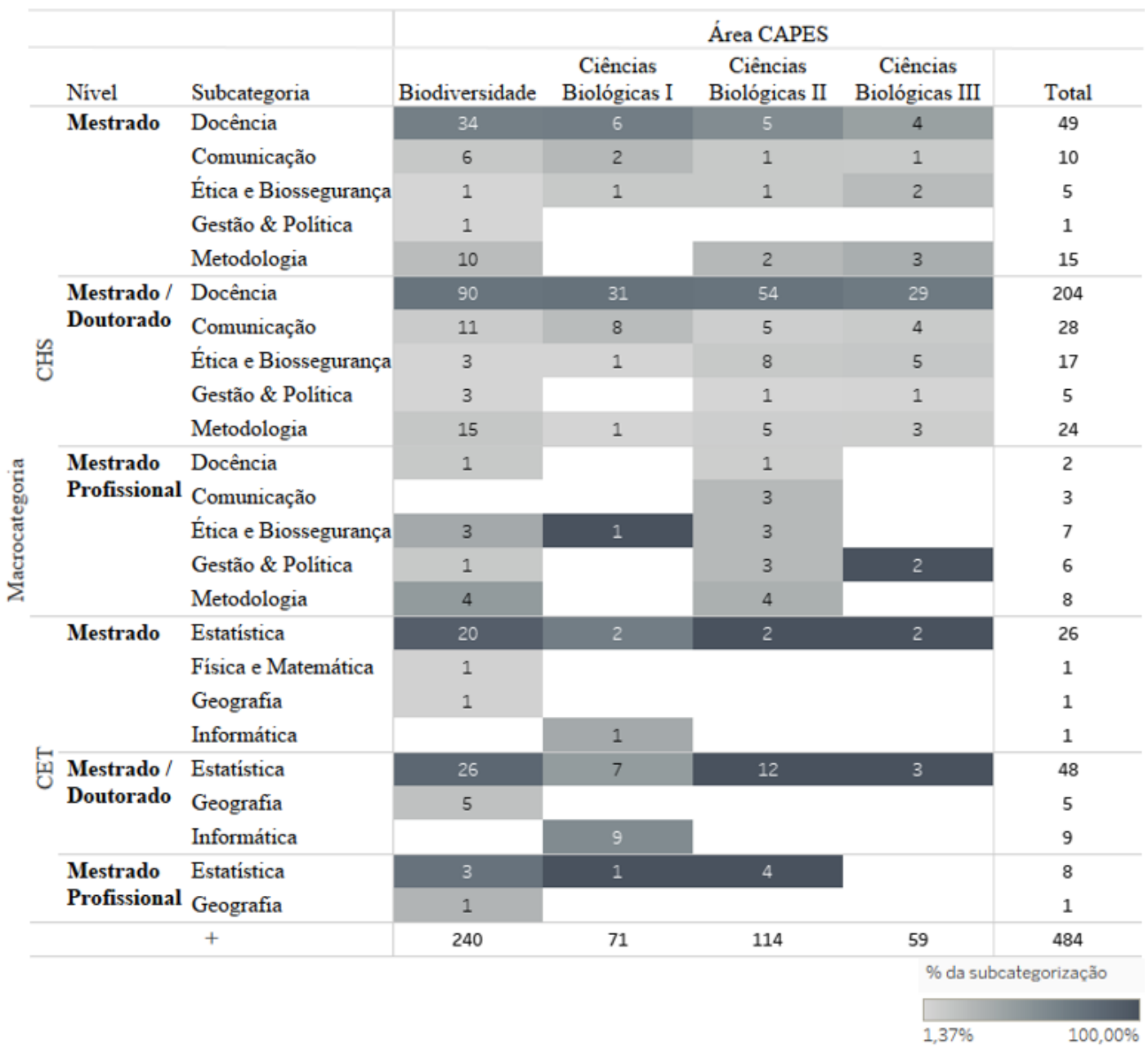

Fonte: Elaborada pelas autoras com dados da Plataforma Sucupira, 2019.

Uma análise mais aprofundada nas ementas das disciplinas de Docência apontou que $75 \%$ das disciplinas oferecidas eram do tipo prática, $22 \%$ do tipo teórica, e apenas $3 \%$ eram teórico-práticas. Ou seja, a maior oferta de disciplinas de ensino e educação era do tipo "estágio para docência", onde o/a discente é o/a responsável por organizar aulas ou um curso específico para a graduação, monitorado ou não por um/a docente.

\section{Considerações finais}

Temas como mudanças climáticas, desastres naturais, acidentes com altos impactos ambientais, consumismo exacerbado, perda de biodiversidade e escândalos éticos na 
biomedicina evidenciam a importância de se formar profissionais com conhecimento e capacidade de atuar de maneira socialmente responsável e confiável na utilização, conservação e recuperação dos recursos naturais. As Ciências Biológicas é uma das grandes áreas que forma pessoal com potencial de atuação em diversos setores estratégicos para o bem-estar da sociedade, como, por exemplo, na educação, meio ambiente, saúde e defesa.

Em diversos países, profissionais que atuam nessa área questionam a capacidade dos currículos das pós-graduações em preparar estudantes para buscar a resolução de problemas locais e em conciliar os objetivos de suas pesquisas com as reais necessidades do mundo (BOSCH, 2018; HORTALE et al., 2014; MORRISON et al., 2011). Também é recorrente a crítica de que nas Ciências Biológicas há um excesso de especialização, com o apoio de currículos que tendem a enfatizar conteúdos que promovem a competência técnica, mas muitas vezes de forma desconectada do contexto social (ANDERSON; ROGAN, 2011; BOSCH, 2018; CLARK, 2001).

No presente trabalho, apresentamos uma análise que permitiu identificar o perfil de todas as disciplinas oferecidas pelos PPGs em Ciências Biológicas no Brasil, no período 20132016. Os resultados forneceram um panorama de distribuição das disciplinas em três grupos temáticos que aglutinam diferentes conteúdos. E, tal como sugerido na literatura internacional, observamos uma enorme ênfase em disciplinas de conteúdo específico das Ciências Biológicas.

As disciplinas de Docência, Estatística e Informática, pertencentes aos grupos de disciplinas com conteúdo não-específico (CHS e CET), foram identificadas com uma frequência maior que as outras deste grupo. Essas duas últimas pertencem a área de conhecimento Ciências Exatas, uma das áreas inseridas na macrocategoria Ciências Exatas e da Terra, utilizada na análise. Tais disciplinas apresentam conteúdos que são muito relevantes para a pesquisa na área de Biodiversidade e Ciências Biológicas I, respectivamente, áreas em que a presença destes tópicos foi mais destacada, o que, aliado a disciplina de Docência nos permite inferir que o currículo oferecido tenderia a contribuir para uma formação mais voltada para a pesquisa e ensino.

Disciplinas que apoiam uma formação mais geral e interdisciplinar (PEREIRA; CARNEIRO; GONÇALVES, 2015), com componentes de humanidades e arte (BARATA, 2019; BOSCH, 2018) e que são importantes para o desenvolvimento de comportamentos específicos das funções exercidas por cientistas e professores do ensino superior (BOTOMÉ; KUBO, 2002) foram ofertadas em alguns programas das Ciências Biológicas. No entanto, a maioria dessas disciplinas ocorre em número muito pequeno, distribuídas em poucos programas 
e quase sempre como não obrigatórias. Neste grupo de disciplinas, as disciplinas de Docência foram a exceção, estando disponíveis em quase todos os programas analisados.

Cabe destacar que embora a Docência tenha se sobressaído entre as disciplinas não específicas, a maioria delas era do tipo exclusivamente prática, cabendo ao docente responsável pela disciplina o ensino sobre o conteúdo específico da disciplina, bem como do pedagógico, relacionado ao ensino no nível superior. Este fato é amplamente criticado na literatura, visto que a prática é importante, mas não garante o efetivo preparo para o exercício da docência, que exige capacitação própria e específica (NGANGA et al., 2015; PATRUS; LIMA, 2014).

Além disso, a identificação de estruturas curriculares focadas prioritariamente em disciplinas específicas, tão pouco plurais, pode ter grande relação com a não pontuação deste quesito no processo de avaliação conduzido pela CAPES. Como o financiamento das atividades das PGs é muito dependente dos recursos oriundos desta Fundação ou de outras agências, que, por sua vez, tem relação direta com o conceito que o PPG recebe da CAPES, é natural que os programas priorizem os critérios com maior peso na avaliação, que, de forma geral, estão apontando para os produtos gerados por eles, em especial, as publicações em periódicos especializados. Neste contexto, outras iniciativas, como a ampliação e a diversificação da grade curricular, ficam em segundo plano ou simplesmente não são consideradas.

Este estudo constatou que os programas de mestrado e doutorado em Ciências Biológicas, acadêmicos ou profissionais, ofereciam uma formação do tipo especialista, priorizando algum recorte do conhecimento dentro da grande área. Como dito anteriormente, o domínio do conhecimento específico é importante, mas não garante a formação acadêmica e profissional necessária para atuação de um cientista. Mas será que os responsáveis pelos programas têm consciência sobre o seu papel enquanto formadores de profissionais? Será que se preocupam com a efetiva formação de seus discentes? Por outro lado, como será que os discentes das pós-graduações percebem sua formação? Será que identificam lacunas ou excessos na formação? Questões como estas, que mostram que há muito ainda a ser explorado nos ambientes das pós-graduações brasileiras, são motivadoras de estudos em andamento realizados pelas autoras do presente trabalho.

Defendemos que a formação de mestres e doutores, em especial das Ciências Biológicas, deva ser repensada a partir dos desafios atuais, que aponta para a necessidade de se organizar e se disponibilizar um currículo que efetivamente promova a interdisciplinaridade, englobando um conjunto de atividades e disciplinas que possibilitem a formação de profissionais mais críticos, reflexivos e criativos, aptos a produzir conhecimento e tecnologias, bem como saber ensiná-los, divulgá-los e administrá-los. 


\section{Referências}

ANDERSON, Trevor R.; ROGAN, John M. Bridging the educational research-teaching practice gap: Curriculum development, Part 1: Components of the curriculum and influences on the process of curriculum design. Biochemistry and Molecular Biology Education, Winnipeg, v. 39, n. 1, p. 68-76, 2011. Disponível em: https://pubmed.ncbi.nlm.nih.gov/21433259/ . Acesso em: 20 fev. 2020.

ARAÚJO, Christine Veloso Barbosa; SILVA, Viviane Nascimento; DURÃES, Sarah Jane. Processo de Bolonha e mudanças curriculares na educação superior: para que competências? Educacao e Pesquisa, São Paulo, v. 44, p. 1-18, 2018. Disponível em: https://www.scielo.br/pdf/ep/v44/1517-9702-ep-44-e174148.pdf . Acesso em: 20 fev. 2020.

BALBACHEVSKY, Elizabeth; SCHWARTZMAN, Simon. The graduate foundations of research in Brazil. Higher Education Forum, Hiroshima, v. 7, n. 1, p. 85-100, 2010.

BALZAN, Newton Cesar. Teses e dissertações: a qualidade em questão. Desdobramentos. Avaliação, Campinas; Sorocaba, v. 17, n. 3, p. 827-849, 2012. Disponível em: https://www.scielo.br/pdf/aval/v17n3/a11v17n3.pdf . Acesso em: 20 fev. 2020.

BARATA, Rita Barradas. Mudanças necessárias na avaliação da pós-graduação brasileira. Interface: comunicação, saúde, educação, Botucatu, v. 23, p. 1-6, 2019. Disponível em: https://www.scielo.br/scielo.php?pid=S1414-32832019000100101\&script=sci_arttext . Acesso em: 20 fev. 2020.

BARDIN, Laurence. Análise de conteúdo. Lisboa: Edições 70, 2006.

BOSCH, Gundula. Train PhD students to be thinkers not just specialists. Nature, Berlim, v. 554, n. 7692, p. 277, 2018. Disponível em: https://www.nature.com/articles/d41586-01801853-1. Acesso em: 20 fev. 2020.

BOTOMÉ, Sílvio Paulo; KUBO, Olga Mitsue. Responsabilidade social dos programas de Pós-graduação e formação de novos cientistas e professores de nível superior. Interação em Psicologia, Curitiba, v. 6, n. 1, p. 1-29, 2002. Disponível em:

https://revistas.ufpr.br/psicologia/article/view/3196 . Acesso em: 20 fev. 2020.

BRYMAN, Alan. Social research methods. New York: Oxford University Press, 2016.

CAPES - COORDENAÇÃO DE APERFEIÇOAMENTO DE PESSOAL DE NÍVEL SUPERIOR. Plano Nacional de Pós-Graduação ( PNPG ) 2011-2020: Documentos setoriais. Brasília, 2010. v. 2. Disponível em:

https://www.capes.gov.br/images/stories/download/PNPG_Miolo_V2.pdf. Acesso em: 20 fev. 2020.

CASNER-LOTTO, Jill; BARRINGTON, Linda. Are they really ready to work? Employers' perspectives on the basic knowledge and applied skills of new entrants to the 21st Century US workforce. New York: The Conference Board, 2006. Disponível em:

https://files.eric.ed.gov/fulltext/ED519465.pdf . Acesso em: 21 fev. 2020.

CERETTA, Carlos Alberto; ANJOS, Lucia Helena Cunha; SIQUEIRA, José Oswaldo. A pós- 
graduação em Ciência do Solo no Brasil: evolução e tendência. RBPG. Revista Brasileira de Pós-Graduação, Brasília, v. 5, n. 9, p. 7-35, 2008. Disponível em:

http://ojs.rbpg.capes.gov.br/index.php/rbpg/article/view/140 . Acesso em: 20 fev. 2020.

CLARK, Tim. Developing policy-oriented curricula for conservation biology: Professional and leadership education in the public interest. Conservation Biology, New Jersey, v. 15, n. 1, p. 31-39, 2001. Disponível em: https://www.jstor.org/stable/2641643?seq=1 . Acesso em: 20 fev. 2020.

DE MEIS, Leopoldo et al. The growing competition in Brazilian science: rites of passage, stress and burnout. Brazilian Journal of Medical and Biological Research, Ribeirão Preto, v. 36, n. 9, p. 1135-1141, 2003. Disponível em:

https://www.scielo.br/scielo.php?script=sci_arttext\&pid=S0100-879X2003000900001 .

Acesso em: 20 fev. 2020.

GALDINO, Maria José Quina et al. Síndrome de Burnout entre mestrandos e doutorandos em enfermagem. Acta Paulista de Enfermagem, São Paulo, v. 29, n. 1, p. 100-106, 2016.

Disponível em: https://www.scielo.br/scielo.php?script=sci_abstract\&pid=S010321002016000100100\&lng=pt\&nrm=iso\&tlng=pt . Acesso em: 20 fev. 2020

GHENO, Ediane Maria et al. Sistema de avaliação da CAPES: indicadores e procedimentos de monitoramento e avaliação de desempenho. Em Questão, Porto Alegre, v. 25, n. 3, p. 184-213, 2019. Disponível em: https://seer.ufrgs.br/EmQuestao/article/view/86490 . Acesso em: 20 fev. 2020.

HORTALE, Virginia Alonso et al. Professional paths of alumni from doctorate programs in health and biological sciences. Revista de Saude Publica, São Paulo, v. 48, n. 1, p. 1-9, 2014. Disponível em: https://pubmed.ncbi.nlm.nih.gov/24789631/ . Acesso em: 20 fev. 2020.

HOSTINS, Regina Célia Linhares. Formação de pesquisadores em programas de excelência de pós-graduação em educação. Revista Brasileira de Educacao, Rio de Janeiro, v. 18, n. 53, 2013. Disponível em: https://www.scielo.br/pdf/rbedu/v18n53/10.pdf . Acesso em: 20 fev. 2020.

KISGEN, Stefanie. The future of business leadership education in tertiary education for graduates. Stuttgart: Steinbeins-Edition, 2017.

LOUZADA, Rita de Cássia Ramos; SILVA FILHO, João Ferreira da. Formação do pesquisador e sofrimento mental: um estudo de caso. Psicologia em Estudo, Maringá, v. 10, n. 3, p. 451-461, 2005. Disponível em: scielo.br/pdf/pe/v10n3/v10n3a12.pdf . Acesso em: 20 fev. 2020.

MARIATT, Dostoiewski; CHAMPANGNATTE, De Oliveira. Currículo universitário: do México ao Brasil neoliberais. Avaliação, Campinas; Sorocaba, v. 21, n. 1, p. 109-123, 2016. Disponível em: https://www.scielo.br/pdf/aval/v21n1/1414-4077-aval-21-01-00109.pdf . Acesso em: 20 fev. 2020.

MEADOWS, Arthur Jack. Communication in science. London: Butterworths, 1974.

MELLO, Alex Fiúza de; ALMEIDA FILHO, Naomar de; RIBEIRO, Renato Janine. Por uma 
universidade socialmente relevante. Atos de Pesquisa em Educação, Blumenal, v. 4, n. 3, p. 292-302, 2009. Disponível em:

https://proxy.furb.br/ojs/index.php/atosdepesquisa/article/view/1718 . Acesso em: 20 fev. 2020.

MISNI, Farahana; NIK MAHMOOD, Nik Hasnaa; JAMIL, Rossilah. The effect of curriculum design on the employability competency of Malaysian graduates. Management Science Letters, Vancouver, v. 10, n. 4, p. 909-914, 2020. Disponível em: http://growingscience.com/beta/msl/3530-the-effect-of-curriculum-design-on-theemployability-competency-of-malaysian-graduates.html . Acesso em: 20 fev. 2020.

MOREIRA, Maria Lígia; VELHO, Lea. Pós-graduação no Brasil: da concepção “ofertista linear" para "novos modos de produção do conhecimento" implicações para avaliação. Avaliação, Campinas; Sorocaba, v. 13, n. 3, p. 625-645, 2008. Disponível em: https://www.scielo.br/pdf/aval/v13n3/02.pdf . Acesso em: 20 fev. 2020.

MORRISON, Emory et al. What Matters for Excellence in PhD Programs? The Journal of Higher Education, Columbus, v. 82, n. 5, p. 535-563, 2011. Disponível em: https://www.tandfonline.com/doi/abs/10.1080/00221546.2011.11777217?tab=permissions\&s croll=top\& . Acesso em: 20 fev. 2020.

NETTO, Carlos Alexandre. Princípios para um novo modelo de avaliação da pós-graduação. Ciência e Cultura, São Paulo, v. 70, n. 3, p. 47-51, 2018. Disponível em: http://cienciaecultura.bvs.br/pdf/cic/v70n3/v70n3a12.pdf . Acesso em: 20 fev. 2020.

NGANGA, Camilla Soueneta Nascimento et al. Mestres e Doutores em Contabilidade no Brasil: Uma Análise dos Componentes Pedagógicos de sua Formação Inicial. REICE. Revista Iberoamericana sobre Calidad, Eficacia y Cambio en Educación, Madrid, v. 14, n. 1, 2015. Disponível em: https://revistas.uam.es/index.php/reice/article/view/2709/2936 . Acesso em: 20 fev. 2020.

PATRUS, Roberto; LIMA, Correa Manolita. A formação de professores e de pesquisadores em Administração: contradições e alternativas. Revista de Economia e Gestão, Belo Horizonte, p. 4-29, 2014. Disponível em: http://periodicos.pucminas.br/index.php/economiaegestao/article/view/6982 . Acesso em: 20 fev. 2020 .

PEREIRA, Elisabete M. A.; CARNEIRO, Ana Maria; GONÇALVES, Mirian Lúcia. Inovação e avaliação na cultura do ensino superior brasileiro: formação geral interdisciplinar. Avaliação, Campinas; Sorocaba, v. 20, n. 3, p. 717-739, 2015. Disponível em: https://www.scielo.br/pdf/aval/v20n3/1414-4077-aval-20-03-00717.pdf . Acesso em: $20 \mathrm{fev}$. 2020.

SILVA, Lidiane Cristina Da et al. Perfil dos programas de pós-graduação Stricto Sensu em Gestão do Conhecimento no Brasil e seu panorama da produção científica. Avaliação, Campinas; Sorocaba, v. 24, n. 1, p. 328-351, 2019. Disponível em: https://www.scielo.br/pdf/aval/v24n1/1982-5765-aval-24-01-328.pdf . Acesso em: 20 fev. 2020.

SOUZA, Saulo Aparecido; REINERT, José Nilson. Participação discente nas publicações da 
Pós-Graduação: um estudo comparativo entre programas públicos e privados de Administração. Avaliação, Campinas; Sorocaba, v. 17, n. 2, p. 481-502, 2012. Disponível em: https://www.scielo.br/pdf/aval/v17n2/10.pdf . Acesso em: 20 fev. 2020.

SPATTI, Ana Carolina; SERAFIM, Milena Pavan; DIAS, Rafael de Brito. Universidade e pertinência social: alguns apontamentos para reflexão. Avaliação, Campinas; Sorocaba, v. 21, n. 2, p. 341-360, 2016. Disponível em: https://www.scielo.br/pdf/aval/v21n2/1982-5765-aval21-02-00341.pdf . Acesso em: 20 fev. 2020.

TAYLOR, Mark C. Reform the PhD system or close it down. Nature, Berlim, v. 472, n. 7343, p. 261, 2011. Disponível em: https://www.nature.com/articles/472261a . Acesso em: 20 fev. 2020. 